\title{
Stability of Semiconductor Memory Characteristics in a Radiation Environment
}

\author{
I. Fetahović, M. Vujisić, K. Stanković, E. Dolićanin \\ Paper dedicated to memory of Vera Nikolić-Stanojević, \\ Pofessor Emeritus at the State University of Novi Pazar
}

\begin{abstract}
Radiation defects in electronic device can occur in a process of its fabrication or during use. Miniaturization trends in industry and increase in level of integration of electronic components have negative affect on component's behavior in a radiation environment. The aim of this paper is to analyze radiation effects in semiconductor memories and to establish how ionizing radiation influences characteristics and functionality of semiconductor memories. Both the experimental procedure and simulation have been used to investigate the behavior of irradiated semiconductor memories. EPROM and EEPROM commercial semiconductor memory samples have been exposed to indirect ionizing radiation to test their radiation hardness. Monte Carlo simulation method has been used to analyze the effect of direct ionizing radiation on semiconductor memory.
\end{abstract}

Keywords: semiconductor memory, radiation effects, simulation

\section{Radiation effects in semiconductor memories}

Disruptions of memory characteristics after its exposure to radiation depend on several factors: type of radiation, speed of energy deposition, and type of material the semiconductor device is made of. If irradiated semiconductor device needs shorter period of time to recover and restore its functionality, the radiation effect is called transient. Permanent radiation effects occur when the device cannot fully restore functionality due to severe changes in its structure or when resolving needs long period of time [1-4].

Single Event Upsets (SEU) and disruptions caused by high radiation dose are examples of transient radiation effects. These effects only induce soft errors, such as false logic states in digital circuits. After short period of time the transient effect often disappears or can be easily removed, and does not cause destructive changes [5,6].

Manuscript received June 17, 2014. ; accepted October 19, 2014.

I. Fetahović, M. Vujisić, K. Stanković, E. Dolićanin are with the State University of Novi Pazar, Novi Pazar, Serbia. 
Radiation of alpha particles from memory chip package can induce soft errors in memory, but cosmic radiation of light and heavy ions can disrupt semiconductor memories' characteristics, causing both soft and hard errors. Charged carriers induced in irradiated memory generate currents that might change logic states of transistors, thus disturbing data written on the chip. These types of disturbances in memory, induced by radiation, are called transient upsets.

\section{The experiment}

\subsection{Gamma radiation experimental procedure}

The source of radiation used to examine gamma radiation effects on EPROM and EEPROM semiconductor memories was ${ }^{60} \mathrm{Co}$. Number of errors in memory samples has been observed after the samples have been irradiated.

Prior to radiation exposure, memory cells of the samples have been written into logic ' 1 ' state, since this state is more radiation sensitive than the logic ' 0 ' state. The experiment has been performed in steps and the radiation dose has been increased in each step. As a result of irradiation, some memory cells changed its logic state to ' 0 ', and this has been defined as an error. After irradiation, number of errors is determined, being the same as the number of logic ' 0 ' at readout.

Combined uncertainty of measurement results was lower than $10 \%[9,10]$.

\subsection{Simulation of direct ionizing radiation interaction with semiconductor memory}

TRIM software has been used to perform Monte Carlo simulations of interaction between direct ionizing radiation and MOS structure insulator layer. TRIM is open source software (part of SRIM 2008 programming package) made for simulation of ions passing through the material [11].

\section{Results and discussion}

\subsection{Indirect ionizing radiation damage}

The average differential and cumulative change in the number of errors in memory samples versus the absorbed dose have been presented in this chapter. Increase in the absorbed dose has caused increase in the number of errors, thus the functionality of EPROM and EEPROM has decreased. These changes are reversible for EPROM memory samples, in contrast to the changes in EEPROM memories.

No error has been observed when the radiation dose in EPROM samples was below $1160 \mathrm{~Gy}$. Increase in the absorbed dose greater than $1260 \mathrm{~Gy}$ has caused significantly larger number of errors in the samples. The first false logic state for EEPROM memories has occurred for radiation dose of $930 \mathrm{~Gy}$, confirming EEPROMs greater sensitivity on gamma radiation. 


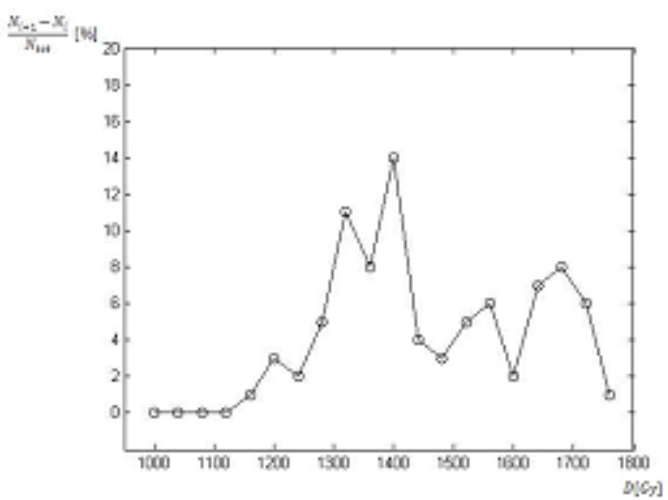

(a)

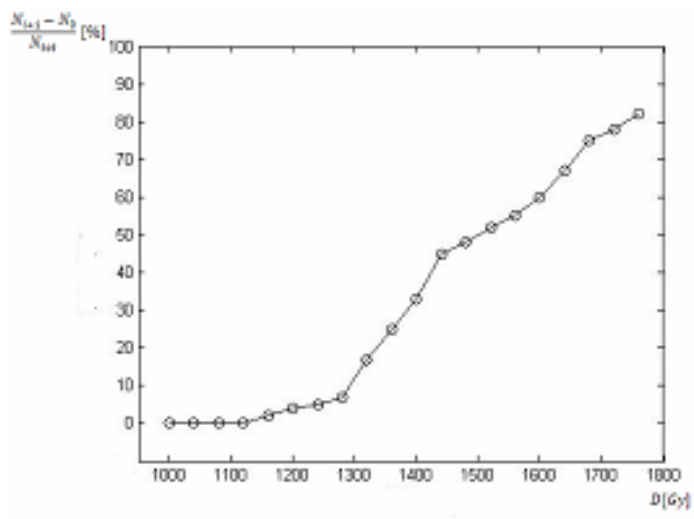

(b)

Fig. 1: The average relative change in number of errors in irradiated EPROM samples (NM27C020) versus the absorbed dose of radiation: a) differential; b) cumulative $\left(\mathrm{N}_{t o t}=2,097,152\right.$ bit, $\left.\mathrm{N}_{0}=0\right)$

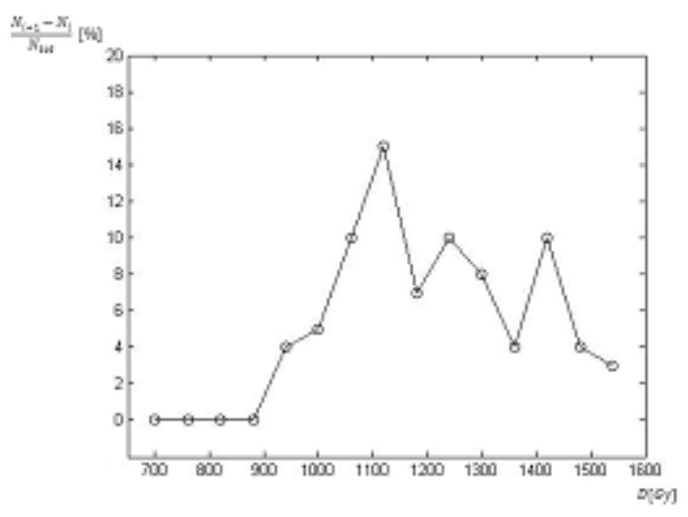

(a)

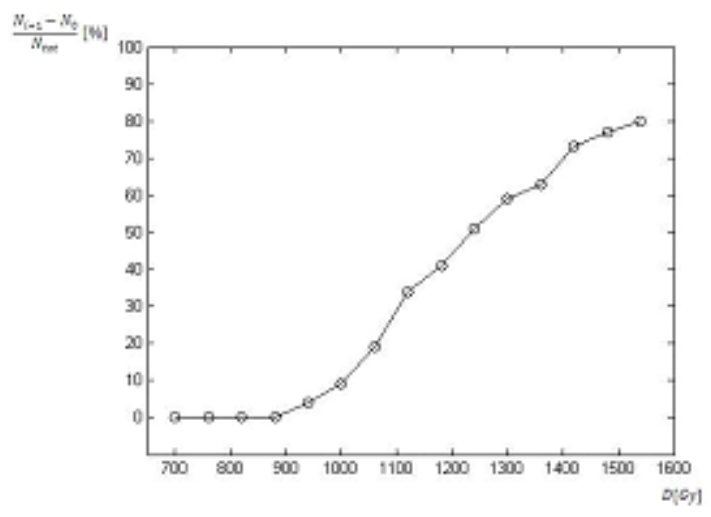

(b)

Fig. 2: The average relative change in number of errors in irradiated EEPROM samples (FM93CS56) versus the absorbed dose of radiation: a) differential; b) cumulative $\left(\mathrm{N}_{t o t}=2048\right.$ bit, $\left.\mathrm{N}_{0}=0\right)$

The main effect caused by gamma radiation of the source ${ }^{60} \mathrm{Co}$ is production of charged particle pairs (electron-hole) in $\mathrm{SiO}_{2}$ insulating layer of the gate. The number of generated pairs depends on the absorbed dose of gamma radiation, material characteristics and available volume of the material. One part of electron-hole pairs is recombined, depending on the intensity of electric field in an irradiated oxide. If the field is greater, larger number of pairs will avoid recombination. The remain of electrons in $\mathrm{SiO}_{2}$ is significantly more mobile than the holes, even at room temperatures. Influenced by the applied voltage, electrons swiftly leave the insulator. One part of holes becomes captured in trapping sites in the oxide, while the remain of holes, influenced by electric field, drift towards the floating gate. They contribute to forming positive charge in the oxide. Part of holes not being captured 
in the oxide is injected in FG, thus decreasing number of electrons in it and decreasing the threshold voltage.

Logical state of a memory cell in both EPROM and EEPROM memories is maintained by the electrical charge stored onto the ?oating gate. This charge determines the value of transistor threshold voltage, making the memory cell ' 0 ' or ' 1 ' at readout.

\subsection{Direct ionizing radiation damage}

The interaction of protons, alpha particles and $\mathrm{Na}^{+}$ions with $1 \mu \mathrm{m}$ thick $\mathrm{SiO}_{2}$ oxide layer has been simulated. The results are presented in figures below.

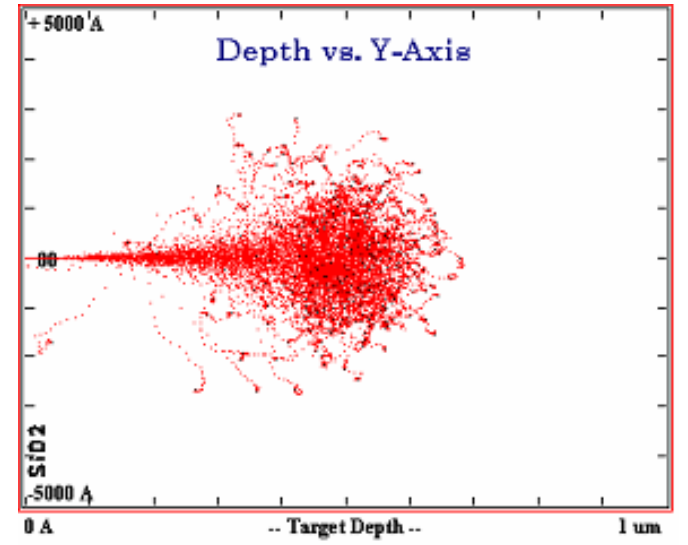

(a)

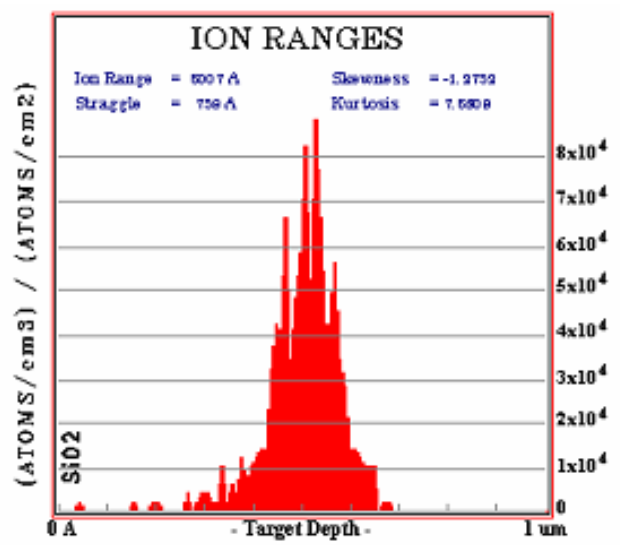

(b)

Fig. 3: The simulation of $50 \mathrm{keV}$ protons passing through $1 \mu \mathrm{m}$ thick layer of $\mathrm{SiO}_{2}(500$ histories $)$ a) Trajectory of protons in $\mathrm{xOy}$ plane $\mathrm{b}$ ) Distribution of stopped protons along the oxide depth

Figures 3a i 4a have confirmed that the penetration depth in target layer is dependent on the energy of a particle. The penetration depth of $100 \mathrm{keV}$ protons in target $\mathrm{SiO}_{2}$ layer is greater than the penetration depth of $200 \mathrm{keV} \mathrm{Na}^{+}$ions since the latter are much heavier. Particle stopping power and specific ionization has decreased per unit path length, as indicated in Figures 3,4,5 and 6.

An incident particle interacts with target atoms in material by scattering on electrons causing ionization, or by elastic scattering on the nuclei. When the energy of a particle transferred to the nucleus is higher than threshold displacement energy, the atom is displaced from its position in a lattice (recoil atom). Along its path, the recoil atoms lose their energy in two ways - by ionization and by displacement of other nuclei. Kinetic energy of a displaced atom can be high enough to cause displacement of other atoms, before it finally occupies interstitial site. Figure $5 \mathrm{~b}$ and $6 \mathrm{~b}$ contain plots depicting energy loss in target layer due to ionization. The ionization is caused by incident particle scattering on target electrons, or as a result of recoil atom colliding with target electrons. Since the alpha particles move much faster than the displaced atoms, the ionization energy loss is greater 


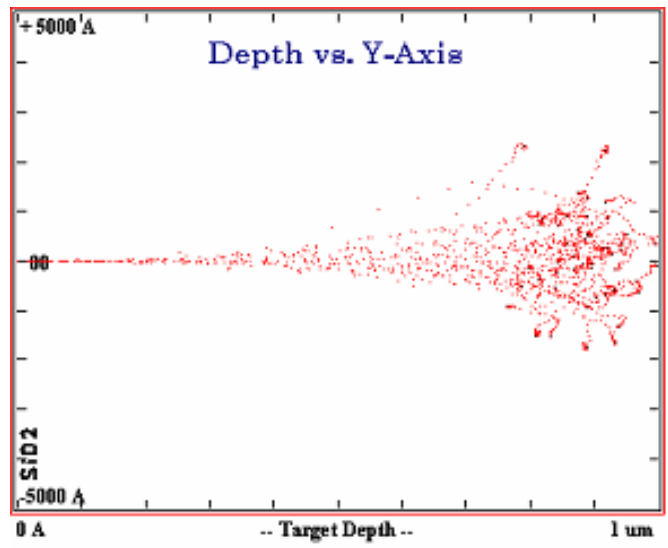

(a)

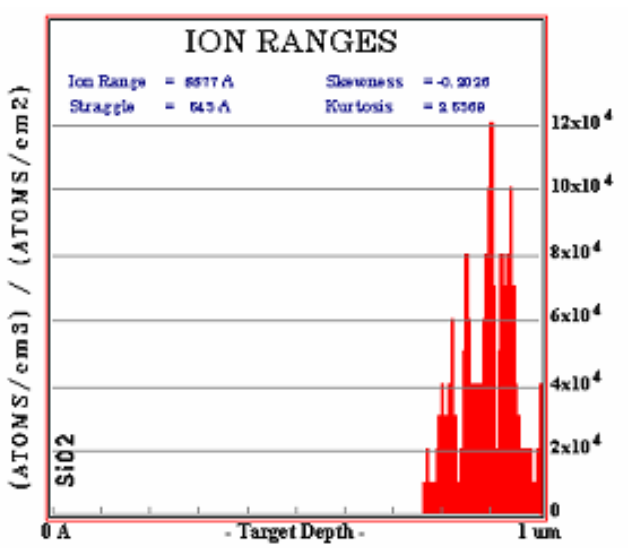

(b)

Fig. 4: The simulation of $100 \mathrm{keV}$ protons passing through $1 \mu \mathrm{m}$ thick layer of $\mathrm{SiO}_{2}(50$ histories) a) Trajectory of protons in $\mathrm{xOy}$ plane b) Distribution of stopped protons along the oxide depth

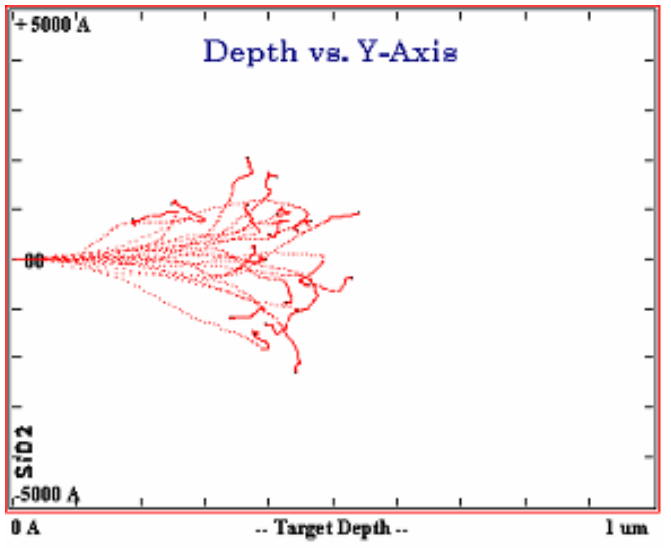

(a)

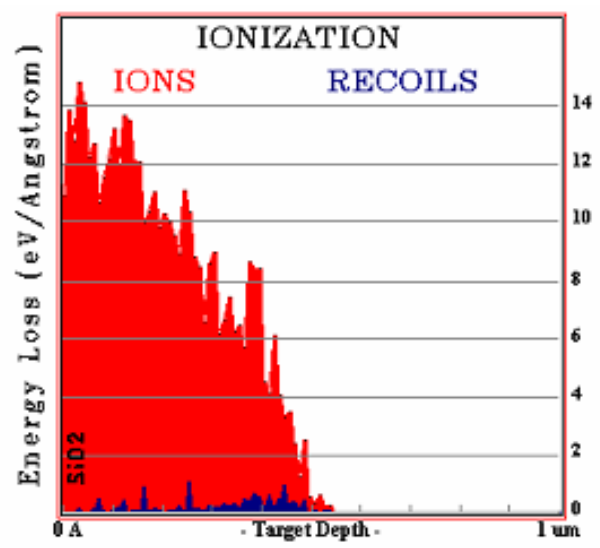

(b)

Fig. 5: The simulation of $50 \mathrm{keV}$ alpha particles passing through $1 \mu \mathrm{m}$ thick layer of $\mathrm{SiO}_{2}$ (20 histories) a) Trajectory of alpha particles in $\mathrm{xOy}$ plane b) The ratio of specific ionization (linear transfer of energy) of incident alpha particles and displaced $\mathrm{Si}$ and $\mathrm{O}$ atoms along the oxide depth

within alpha particles than within recoil atoms, as indicated in Figure 5b. 


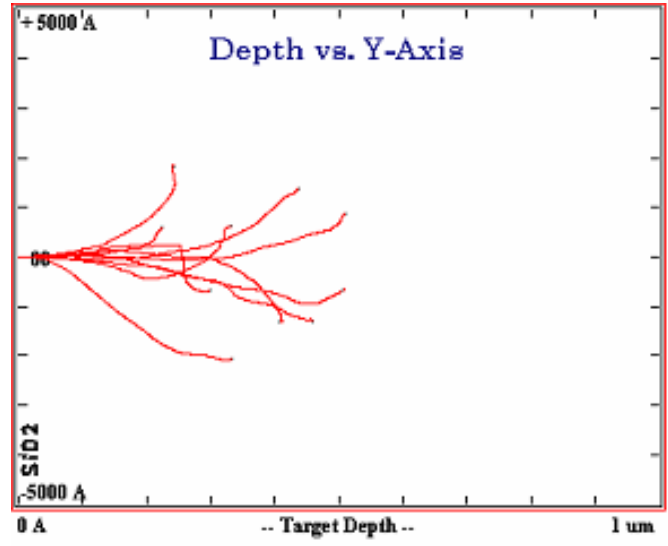

(a)

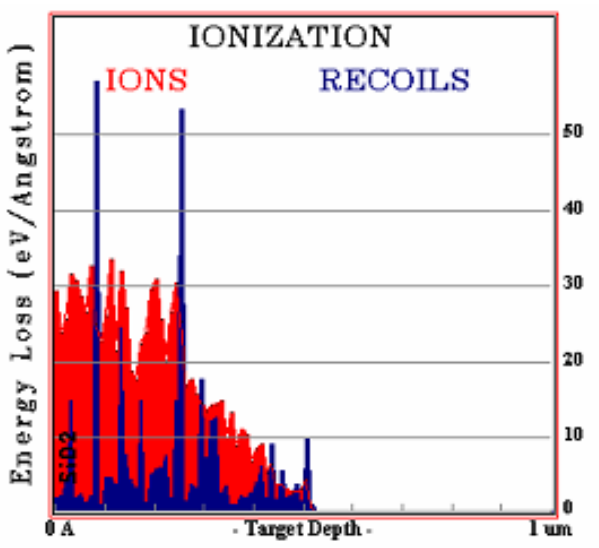

(b)

Fig. 6: The simulation of $200 \mathrm{keV} \mathrm{Na}{ }^{+}$ions passing through $1 \mu \mathrm{m}$ thick layer of $\mathrm{SiO}_{2}(10$ histories $)$ a) Trajectory of $\mathrm{Na}^{+}$ions particles in xOy plane b) The ratio of specific ionization (linear transfer of energy) of incident $\mathrm{Na}^{+}$ ions and displaced $\mathrm{Si}$ and $\mathrm{O}$ atoms along the oxide depth.

\section{Conclusion}

Based on performed experiments and theoretical models of interaction between ionizing radiation and basic semiconductor devices, it can be concluded that there are three basic effects in semiconductor memories exposed to gamma radiation: hole trapping in an oxide, hole injection from oxide into FG and electron emission over the FG/oxide barrier.

Generation of electron-hole pairs leads to holes trapping in an insulator, and electron trapping in insulator/channel interface. Positive charge (hole) trapping causes negative shift in $I_{D} / V_{G}$ characteristics. Negative gate voltage requires accumulation of positively charged carriers on $\mathrm{Si} / \mathrm{SiO}_{2}$ interface, so positively charged carriers induced by ionizing radiation require increase in negative gate voltage to restore balance. Hence, hole trapping and hole injection effects influenced by radiation leads to decrease in threshold voltage. Both of these effects are proportional to the thickness of an oxide. Most of holes appear close to the oxide/substrate or oxide/FG barrier. Positive charge of trapped holes have tendency to mask negative charge on the gate leading to decrease in the FG transistor threshold voltage.

Radiation effects in semiconductor memories are complexly dependent on a material, thickness of an insulating layer, and of methods used to create insulating layer on Si surface. Future research in this area can be directed towards these issues and problems: 1) analyzing the influence of insulating material and thickness of an oxide, 2) considering new methods of creating insulating layer on Si surface, 3) examining change in carrier mobility, 4) studying the effect of dose rate. 


\section{Acknowledgment}

The Ministry of Education, Science and Technological Development of the Republic of Serbia supported this work under contract 171007.

\section{References}

[1] Barth, J.L., Dyer, C.S., Stassinopoulos, E.G., Space, Atmospheric, and Terrestrial Radiation Environments, IEEE Trans. Nucl. Sci., 50 (2003), 3, pp. 466-482

[2] V. S. Vavilov, N. A. Ukhin, Radiation Effects in Semiconductors and Semiconductor Devices, Consultants Bureau, New York, 1977.

[3] R. Radosavljevi, A. Vasi, Effects of Radiation on Solar Cells as Photovoltaic Generators, $\mathrm{Nu}$ clear Technology \& Radiation Protection (2012), vol. 27, No 1, pp. 28-32

[4] D. Nikoli, A. Vasi, I. Fetahovi, K. Stankovi, P. Osmokrovi, Photodiode Behavior in Radiation Environment, Scientific Publications of the State University of Novi Pazar Series A: Applied Mathematics, Informatics \& Mechanics, Vol. 3, No. 1, pp. 27-34, 2011.

[5] G. Holmes-Siedle, L. Adams, Handbook of Radiation Effects, Second Edition, Oxford University Press, 2002.

[6] G. C. Messenger, M. S. Ash, The Effects of Radiation on Electronic Systems, Van Nostrand Reinhold, New York, 1992.

[7] M. Vujisi, N. Marjanovi, I. Fetahovi, K. Stankovi, P. Osmokrovi, Influence of Radiation on Titanium Dioxide Mermistors, Scientific Publications of the State University of Novi Pazar Series A: Applied Mathematics, Informatics \& Mechanics, Vol. 4, No. 1, pp. 75-82, 2012.

[8] M. Zdravkovi, A. Vasi, R. Radosavljevi, M. Vujisi, P. Osmokrovi, Influence of Radiation on the Properties of Solar Cells, Nuclear Technology \& Radiation Protection (2011), vol. 26, No 2, pp. 158-163

[9] K. Stankovi, Influence of the Plain-parallel Electrode Surface Dimensions on the type A Measurement Uncertainty of GM Counter, Nuclear Technology \& Radiation Protection (2011), vol. $26,-1573$

[10] . Dolićanin, K. Stankovi, D. Dolianin, B. Lonar, Statistisal Treatment of Nuclear Counting Results, Nuclear Technology \& Radiation Protection (2011), vol. 26, No. 2, pp. 164-170

[11] Ziegler, J.F., Biersack, J.P., Ziegler, M.D., SRIM (The Stopping and Range of Ions in Matter), Avaliable online: http://www.srim.org

[12] Warner, J.H., Messenger, S.R., Walters, R.J., Summers, G.P., Displacement damage correlation of proton and silicon ion radiation in GaAs, IEEE Trans. Nucl. Sci., 52 (2005), 6, pp. 2678-2682

[13] Vujisic, M., Stankovic, K., Marjanovic, N., Osmokrovic, P., Simulated Effects of Proton and Ion Beam Irradiation on Titanium Dioxide Memristors, IEEE Trans. Nucl. Sci., 57 (2010), 4, pp. 1798-1804

[14] S. Stankovi, B. Irianin, D. Nikoli, K. Jankovi, M. Radenkovi, K. Stankovi, P. Osmokrovi, MSV Signal Processing System for Neutron-Gamma Discrimination in a Mixed Field, $\mathrm{Nu}$ clear Technology \& Radiation Protection, Vol. 27, No. 2, pp. 165-170, (2012) 